\title{
Performance Enhancement in Routing using Leach and SAR Protocol
}

\author{
Prabhdeep Singh \\ Department Of Computer Science \\ Lovely Professional University \\ Phagwara, Punjab, India
}

\author{
Max Bhatia \\ Assistant Professor \\ Department Of Computer Science \\ Lovely Professional University \\ Phagwara, Punjab, India
}

\begin{abstract}
Wireless sensor network are used to monitor the data and collecting the information. Nodes in network sense the data and collect the data then send it to the base station or the sink. However it requires a high power to send data directly to the sink by all the nodes individually so first there are different type of protocols which are discovered to send the data from nodes to the sensors. LEACH operates on two rounds and the set of node at each round is Cluster nodes $(\mathrm{CH})$. At the end of each round each node that is not cluster head select the closest $\mathrm{CH}$ and joins to that cluster head to transmit data. SAR operates for providing the QoS and multiple trees are generated on the basis of energy, QoS and the priority of the packets. The best available path is selected to send the data from nodes to sink or base station. Research is to combine these protocols for reducing overhead of routing tables in SAR and lifetime of the network is increased. The results are compared with the E-LEACH protocol and it is shown that the purposed protocol increase the lifetime of network as compared to E-LEACH.
\end{abstract}

\section{Keywords}

Wireless sensor networks, LEACH, SAR;

\section{INTRODUCTION}

Wireless sensor network are established for collecting the data and monitoring the information by the sensing capabilities of sensor nodes. In WSN sensor nodes sense the data and collect it then send it to the base station. There are number of sensor nodes in a wireless sensor network which was originally developed for military area where human cannot reach or survive to detect the enemy's activity. Sensor nodes are of small size computers which act as both to collect the data and as a router for transferring data. There is one high energy node called as base station which acts as a gateway between wireless sensor network and other outer networks. Due to the small size of nodes, each node has limited memory, power, battery, energy etc. So there are number of protocols designed which are applied in the wireless sensor network so that nodes can communicate for last long periods as possible.[1] Nodes sense data and collect it then send to base station. Base station is end terminal for user to collect data from a wireless sensor network. There are number of application for wireless sensor network such as monitoring application for indoor and outdoor environmental, process automation and structural monitoring are included. Tracking application in wireless sensor network is used to track the objects, humans, vehicles and animals. There are following categories of application in wireless sensor network Disaster management, health application, home applications, military applications, traffic control and explosive material etc. Wireless sensor network structure consist three main components:

$>$ Sensing field- Area where sensor nodes are deployed to gather the information is known as the sensing field

$>$ Sensor nodes- These are the units which gather data and transmit it to base station.

$>$ Target- In sensing field target means the node or area from which data should collect.

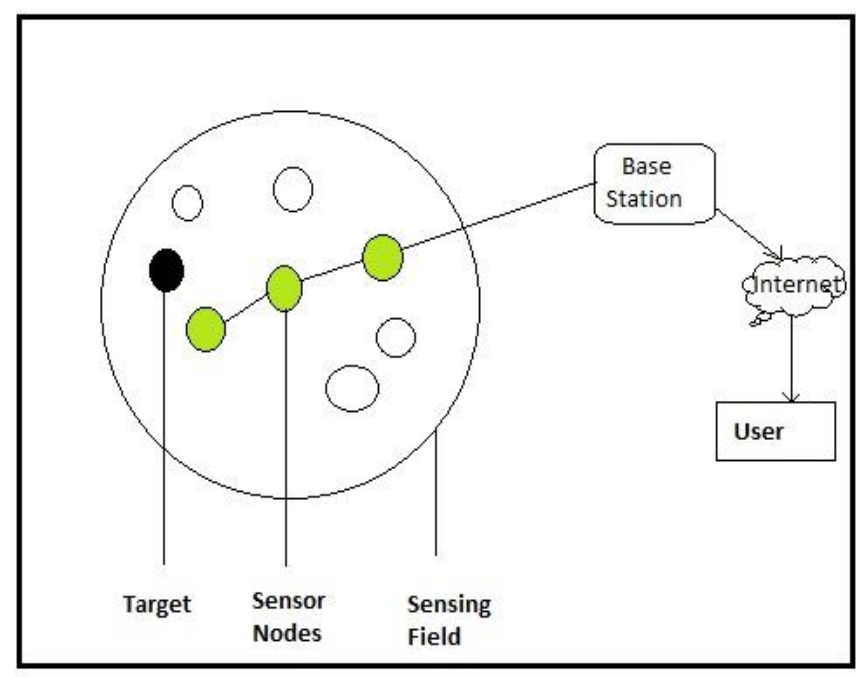

Fig 1. WSN Structure

Due to the small size of sensor nodes it is main issue about use of energy that how battery can be saved for a longer period of time and network lifetime can increase. Due to the batteries with limited energy to recharge the batteries again it is difficult to maintain the sensor nodes due to high cost. [1][2]

\section{CLUSTERING IN WSN}

Energy usage is an important issue in the design of WSNs which typically depends on portable energy sources like batteries for power. Micro-Electro-Mechanical System (MEMS) sensor technology has facilitated the development of smart sensors, these smart sensors nodes are small devices with limited power, processing and computation resources. Smart sensors are power constrained devices that have one or more sensors, memory unit, processor, power supply and actuator. In WSNs, sensor nodes have constrained in term of processing power, communication bandwidth, and storage space which required very efficient resource utilization. In WSNs the sensor nodes are often grouped into individual 
disjoint sets called a cluster, clustering is used in WSNs, as it provides network scalability, resource sharing and efficient use of constrained resources that gives network topology stability and energy saving attributes. Clustering schemes offer reduced communication overheads, and efficient resource allocations thus decreasing the overall energy consumption and reducing the interferences among sensor nodes. A large number of clusters will congest the area with small size clusters and a very small number of clusters will exhaust the cluster head with large amount of messages transmitted from cluster members. [2].

Hierarchical routing is an efficient way to lower energy consumption within a cluster, performing data aggregation and fusion in order to decrease the number of transmitted messages to the BS. Besides achieving energy efficiency, clustering reduces channel contention and packet a collision, resulting in better network throughput under high load. Clustering has been shown to improve network lifetime, a primary metric for evaluating the performance of a sensor network. In addition to supporting network scalability, clustering has numerous advantages. It can localize the route set up within the cluster and thus reduce the size of the routing table stored in the individual node. Clustering can also conserve communication bandwidth since it limits the scope of inter-cluster interactions to $\mathrm{CHs}$ and avoids redundant.[3].

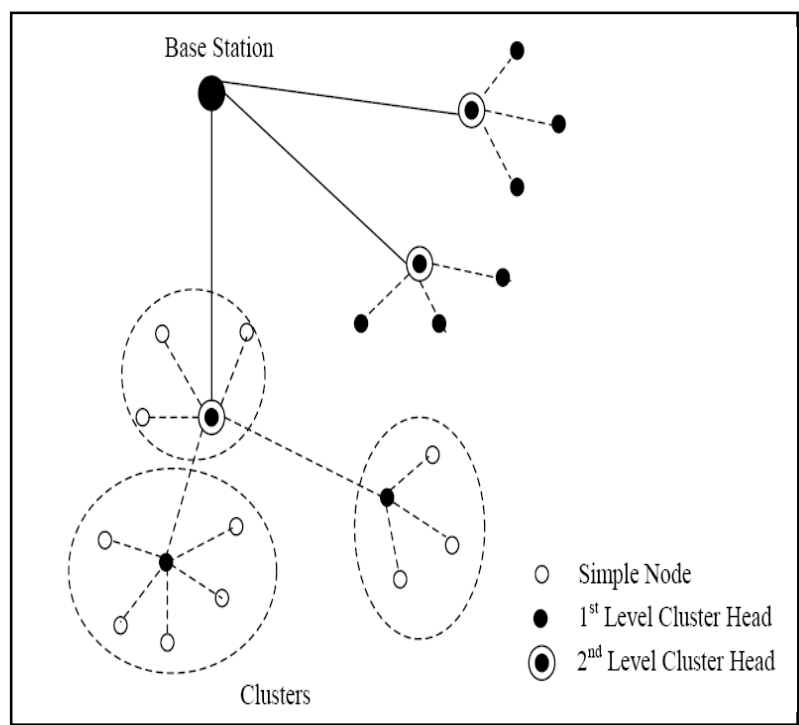

Fig. 2.Clustering in wireless sensor network

The main target of hierarchical routing or cluster based routing is to efficiently maintain the energy usage of sensor nodes by involving them in multi-hop communication within a particular cluster. Clustering plays an important role for energy saving in WSNs. With clustering in WSNs, energy consumption, lifetime of the network and scalability can be improved.. Clustering has important applications in highdensity sensor networks, because it is much easier to manage a set of cluster representatives (cluster head) from each cluster than to manage whole sensor nodes. In WSNs the sensor nodes are resource constrained which means they have limited energy, transmit power, memory, and computational capabilities. Energy consumed by the sensor nodes for communicating data from sensor nodes to the base station is the crucial cause of energy depletion in sensor nodes. [3].

\subsection{LEACH PROTOCOL}

Leach is "Low Energy Adaptive Clustering Hierarchy" protocol which is the first energy efficient routing protocol for hierarchical clustering. Leach is also known as energy efficient routing protocol for clustered wireless sensor networks. Different Clusters are generated for the whole sensor nodes in the network and in each cluster one cluster head is chosen randomly. The nodes with more energy have more chances to become the cluster head. The nodes in each cluster which are not cluster head means non cluster head sense the data and transmit to the cluster head then cluster heads in each cluster aggregate the data and send it to the base station. This operation performed for number of rounds and after each round cluster head are changed. The cluster heads which become the cluster head for once it cannot become the cluster for the next $\mathrm{p}$ rounds where $\mathrm{p}$ is the probability of becoming a cluster head. Each node has a $1 / p$ probability to become cluster head in each round.

There are two phases in Leach Protocol which are as follows:-

$>$ Setup Phase: In the setup phase, a sensor node selects random number between 0 and 1 . If this number is less than the threshold $T(n)$, the node becomes a CH. $T(n)$ is computed as:

$$
T(n)=\left\{\begin{array}{lr}
p / 1-p^{*}(\operatorname{rmod} 1 / p) & \text { if } n \varepsilon g \\
0 & \text { otherwise }
\end{array}\right.
$$

$r$ is the current round; $p$, the desired percentage for becoming $\mathrm{CH}$; and $G$ is the collection of nodes not elected as a $\mathrm{CH}$ in the last $1 / p$ rounds.

Each node decides independent of other nodes if it will become a $\mathrm{CH}$ or not. This decision takes into account when the node served as a $\mathrm{CH}$ for the last time (the node that hasn't been a $\mathrm{CH}$ for long time is more likely to elect itself than nodes that have been a $\mathrm{CH}$ recently). In the following advertisement phase, the $\mathrm{CHs}$ inform their neighborhood with an advertisement packet that they become CHs..After that steady-state phase.[4].

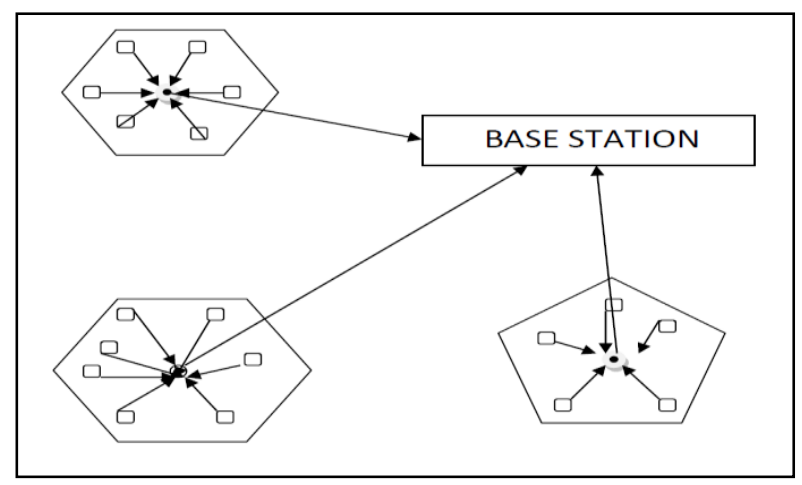

Fig.3. LEACH PROTOCOL

$>$ Steady-State Phase: Data transmission begins; Nodes send their data during their allocated TDMA slot to the $\mathrm{CH}$. This transmission uses a minimal amount of energy (chosen based on the received strength of the $\mathrm{CH}$ advertisement). The radio of each non- $\mathrm{CH}$ node can be turned off until the nodes allocated TDMA slot, thus minimizing energy dissipation in these nodes. When all the data has been received, the $\mathrm{CH}$ aggregate these data and send it to the BS. [4] [5] 


\subsection{SAR}

SAR stands for sequential assignment routing. Sequential assignment routing create the multiple trees from the root where roots are the neibhours of the base station and base station makes the one hop links with the neibhouring nodes. Then tree is generated outward in the network from these nodes and avoid the nodes with low throughput and high delay. When this procedure completes there are the number of nodes which belongs to multiple trees then they choose one tree for send data to base station through one root which is neighbor of base station.

SAR protocol is a multipath routing protocol which makes the routing decision on the base of three factors: energy resources, QoS on each path and packet priority level. The numbers of available paths are discovered among the nodes to the sink node. Among the entire paths best path is chosen for sending the data and best path provide the lower delay with high throughput. The priority level of the packet is considered to choose the paths which are available.

There are two parameters are assigned with each sensor node about each path through it:

$>$ The available energy resources on the path.

$>$ Additive quality of service metric.

The SAR chooses a path which has the high estimated energy resources and a weighted QoS metric is used to handle prioritized packets which are computed as a product of priority level and delay. So the higher priority packets choose the lower delay paths and lower priority packets have to choose the path with higher delay.[6]

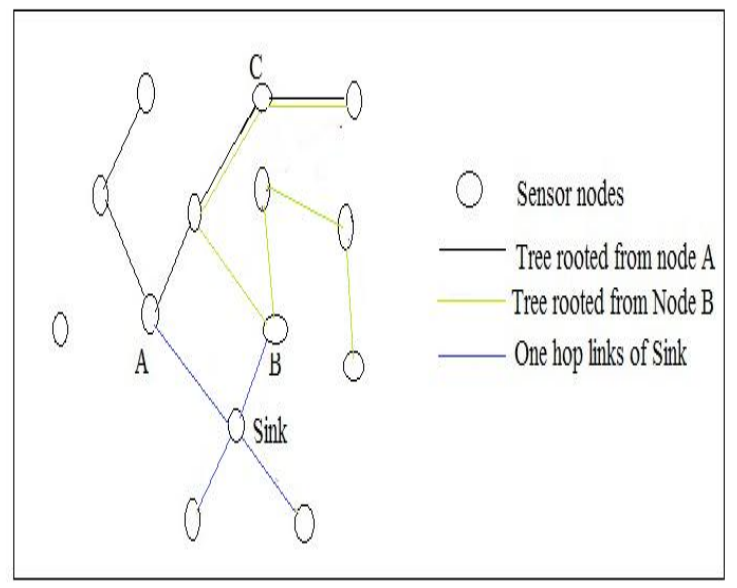

Fig.4. SAR PROTOCOL

\section{PROPOSED MODEL}

E-LEACH is the energy leach protocol. In the E-LEACH protocol network lifetime is increased as compare to LEACH. To increase the lifetime time of network the concatenate of LEACH and SAR is proposed on this paper for increasing the lifetime of network. The purposed model consists of following steps:

\subsection{Deployment of sensor nodes}

Network is taken as homogeneous in which energy of all nodes is same and there are 100 numbers of nodes in the network. Energy of the nodes is represented as E. all nodes have the common energy.

\subsection{Cluster formation and election of cluster head}

The nodes are grouped together to form the clusters. After the formation of clusters, the cluster heads are elected in each and every cluster. Leach choose the cluster head in each cluster and aggregate data in each cluster from other nodes to cluster head then cluster heads transmit data to the base station.

\subsection{Data transmission}

All the nodes in the particular cluster transmit the data to cluster head. SAR protocol discovers the numbers of available paths are discovered among the nodes to the sink node. Among the entire paths best path is chosen for sending the data and best path provide the lower delay with high throughput. In the proposed protocol by using routing firstly LEACH Protocol will apply on the nodes i.e. After that cluster head will generate but not send data to the base station then by applying SAR Protocol on the cluster heads and multiple trees are generated on the basis of priority of the packets. Among all the paths best path is selected and cluster head will send the data to sink among the cluster heads and then the data will send to base station.

By applying firstly leach on the nodes it will generate the cluster heads and all the sensor nodes in each cluster send data to cluster heads and then applying the SAR algorithm on the Cluster heads one hop link is created with neibhouring cluster heads of the base station and then multiple trees are generated outward from these nodes in the network. Then number of cluster heads are connected with number of trees and cluster heads will choose the one tree to send data to base station through one root of neibhouring cluster heads of base station.. In this by using both algorithms SAR and LEACH will give Energy efficiency to the nodes. This will improve lifetime of the network.

\subsection{Energy Model}

Let, there is $\mathrm{m}$ bit of data has to be transmitted. Following equation are used to measure the amount of energy consumption by nodes to send the $m$ bit data to the distance dis. The given equation is known as the multipath fading model and this equation is used when the value of dis is such that:-

dis $>$ dis $_{0}$

$$
\mathrm{E}_{\mathrm{td}-\mathrm{tc}}=\mathrm{m} *\left(\mathrm{E}_{\mathrm{td}}+\mathrm{E}_{\mathrm{a}} \mathrm{d}^{4}\right)
$$

Energy consumed for transmission is

$$
\mathrm{E}_{\mathrm{td}-\mathrm{tc}}
$$

Energy for the transmission of the data is

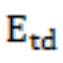

The amount of energy for the amplification is

The amount of energy consumed during the reception of $\mathrm{k}$ bit of data is calculated from the equation given above. The energy consumed during the reception is represented such as:

To measure the amount of energy consumption by nodes to receive the $m$ bit data is calculated using the equation $\mathrm{E}_{\mathrm{rd}-\mathrm{tc}}$

\section{SIMULATION RESULTS}

MATLAB simulation tool is used to simulate the results using LEACH and SAR. Under this section simulations are used to analyze the performance of two protocols combining to increase the lifetime of network. The results calculated using 
combined protocols is compared with the LEACH and ELEACH results.

\subsection{Simulation Step}

There are 100 numbers of nodes which are deployed randomly in the area. Energy of all the nodes is 0.5 initially. Each node sends $m$ bits of data to cluster head in each round. The data packet is of the size 4000 bits. Here the Table 1 describes all the parameters used in the process of simulation. The table is shown below:

Table 1.Simulation parameters

\begin{tabular}{|l|l|l|}
\hline Description & Symbol & Value \\
\hline Number of nodes & $\mathrm{n}$ & 100 \\
\hline Initial energy node & $\mathrm{E}$ & $0.5 \mathrm{~J}$ \\
\hline $\begin{array}{l}\text { Energy consumed by } \\
\text { the amplifier to transmit } \\
\text { the data }\end{array}$ & $\mathrm{E}_{\mathrm{a}}$ & $0.0013 \mathrm{pJ} / \mathrm{bit} / \mathrm{m} 4$ \\
\hline $\begin{array}{l}\text { Energy required for the } \\
\text { transmission of the } \\
\text { signal }\end{array}$ & $\mathrm{E}_{\mathrm{td}}$ & $50 \mathrm{pJ} / \mathrm{bit}$ \\
\hline $\begin{array}{l}\text { Energy required for the } \\
\text { reception of the signal }\end{array}$ & $\mathrm{E}_{\mathrm{rd}}$ & $50 \mathrm{pJ} / \mathrm{bit}$ \\
\hline Data packet aggregation & $\mathrm{E}_{\mathrm{da}}$ & $5 \mathrm{pJ} / \mathrm{bit} / \mathrm{report}$ \\
\hline $\begin{array}{l}\text { Data } \\
\text { energy }\end{array}$ & $\mathrm{M} * \mathrm{M}$ & $100 \mathrm{~m} * 100 \mathrm{~m}$ \\
\hline Sensing Area & $\mathrm{p}$ & 0.05 \\
\hline $\begin{array}{l}\text { Cluster probability of } \\
\text { LEACH }\end{array}$ & \\
\hline
\end{tabular}

\subsection{Simulations and analysis}

Hundred nodes are deployed randomly in the area of $100 * 100 \mathrm{~m}$ square meters and base station is located at the position $(50,140)$.

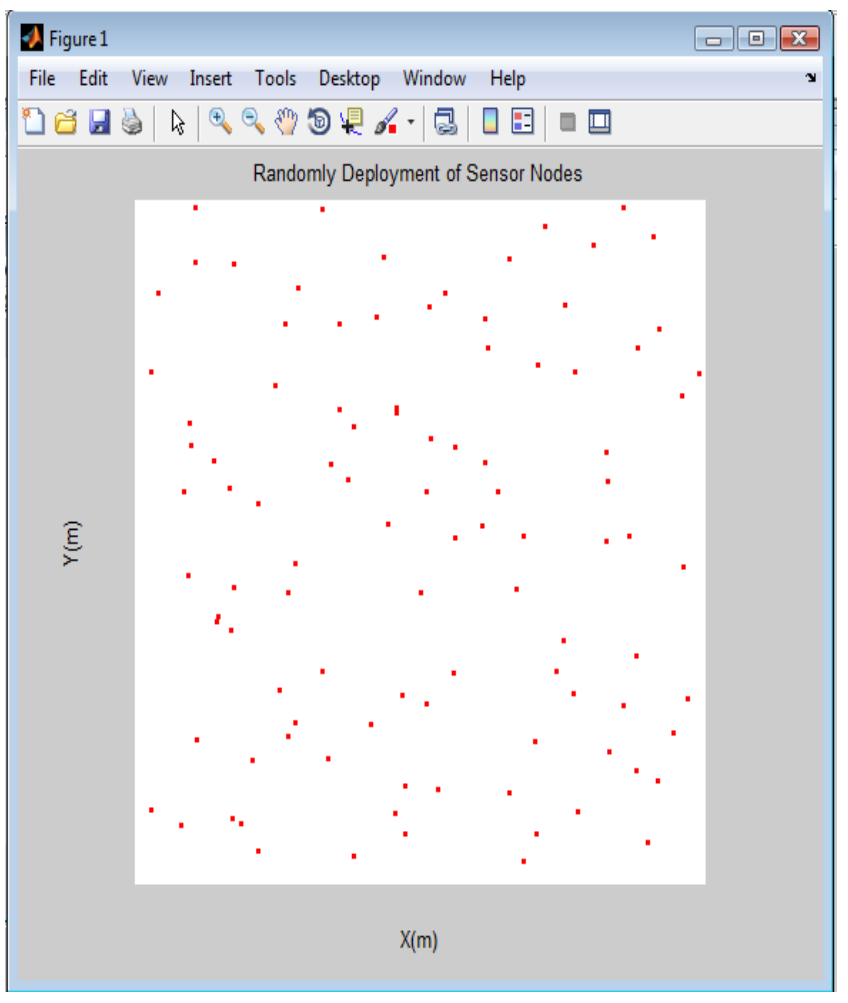

Fig. 5 Snapshot of sensor node deployment
The nodes are grouped together to form the clusters. The cluster formation is shown below:

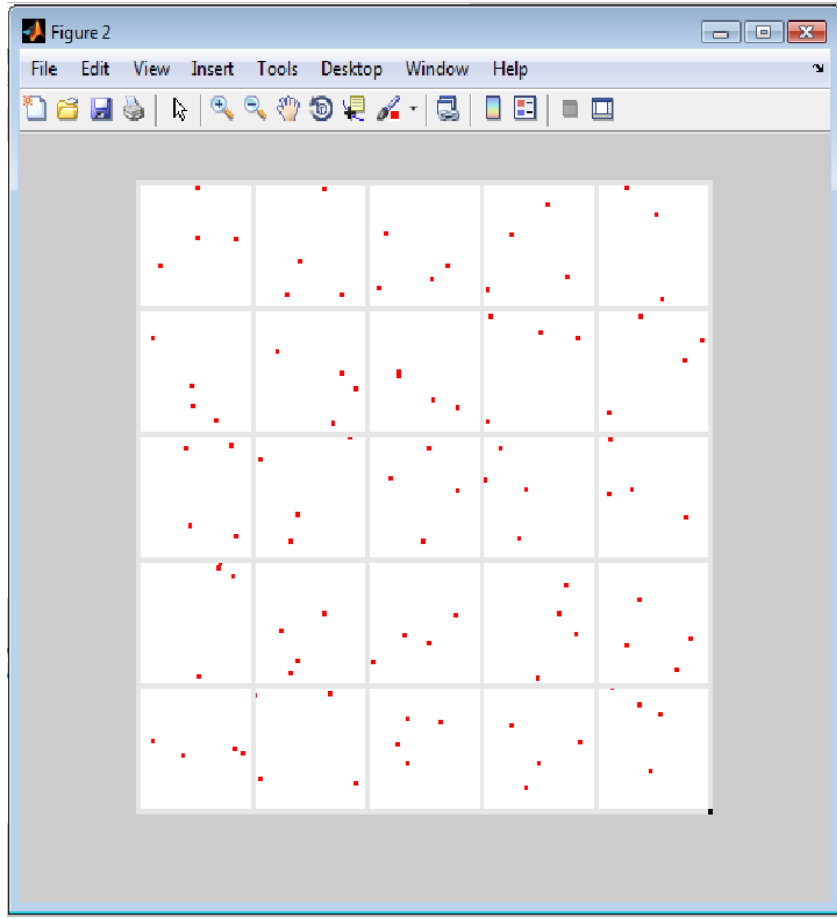

Fig.6. Cluster formation

The leach is applied to elect the cluster head and then SAR is applied on the cluster heads for the transmission of cluster heads to sink. Here the sink is represented by blue box.

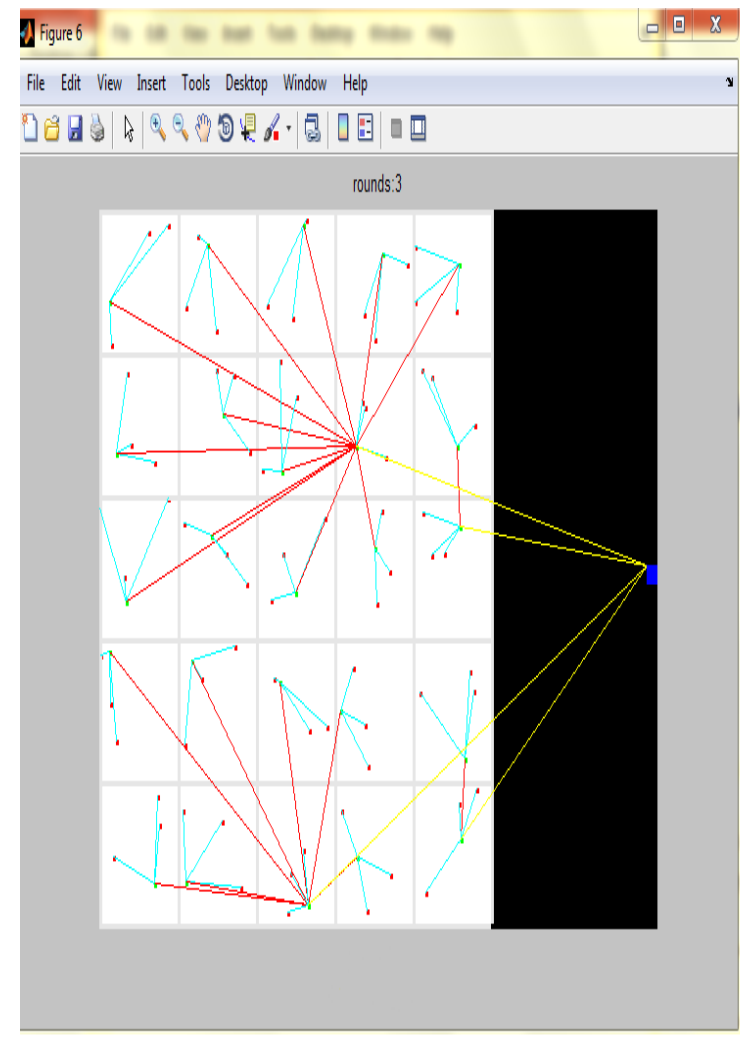

Fig.7. Snapshot of purposed model

The increased network lifetime by the purposed protocol is shown below: 


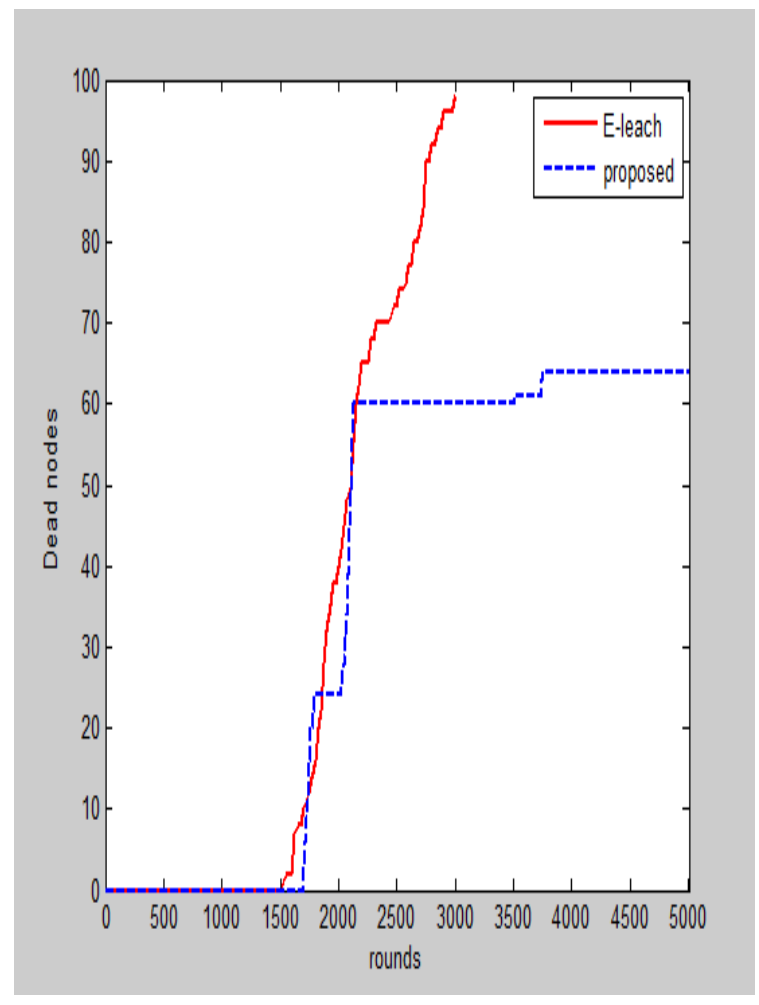

Fig. 8 Number of nodes dead with rounds

To validate the results the purposed protocol is compared with the E-Leach protocol. In the E-LEACH protocol the first node dies on 1529(FND) round and last node dies (LND) on 2082. But in the proposed model the first node dies on 1659 round and last node dies on 2226 round. This increases the lifetime of network as compared to E-LEACH. The comparison is shown in given below table:

Table 2. Comparison of FND and HND

\begin{tabular}{|l|l|l|}
\hline Protocol & FND & HND \\
\hline E-LEACH & 1529 & 2082 \\
\hline Purposed & 1659 & 2226 \\
\hline
\end{tabular}

\section{CONCLUSION}

There are number of protocol for energy saving in wireless sensor network. In this paper we proposed the algorithm combining LEACH and SAR which increase the lifetime of network as compare to LEACH and E-LEACH both. By using the proposed protocol first node dies at 1659 round which is later than E-LEACH.

As in wireless sensor network lifetime of sensor nodes play an important role in performance of network. Energy consumption and throughput are important parameter of QoS in the network. In this dissertation both are enhanced using LEACH and SAR but in future using another protocols such as combination of PEGASIS, EAR etc can enhance in network lifetime of wireless sensor network. Routing path can be optimized using other protocols.

\section{REFERENCES}

[1] Farizah Yunus, Nor-Syahidatul N. Ismail, Sharifah H. S. Ariffin, A. A. Shahidan, Norsheila Fisal, Sharifah K. Syed- Yusof "Proposed Transport Protocol for Reliable Data Transfer in Wireless Sensor Network (WSN)" 2011 IEEE.

[2]. Chompunut Jantarasorn, Chutima Prommak "Minimizing Energy Consumption in Wireless Sensor Networks using Binary Integer Linear Programming" International Journal of Computer and Communication Engineering 6 2012.

[3]. Vinay Kumar, Sanjeev Jain and Sudarshan Tiwari "Energy Efficient Clustering Algorithms in Wireless Sensor Networks: A Survey" IJCSI International Journal of Computer Science Issues, Vol. 8, Issue 5, No 2, September 2011.

[4]. Chenmin Li , Guoping Tan , Jingyu Wu, Zhen Zhang, Lizhong $\mathrm{Xu}$ "Analyzing Cluster-head Selection Mechanisms and Improving the LEACH "2011 IEEE.

[5]. M. Bani Yassein, A. Al-zou'bi, Y. Khamayseh, W. Mardini "Improvement on LEACH Protocol of Wireless Sensor Network (VLEACH)" International Journal of Digital Content Technology and its Applications Volume 3, Number 2, June 2009.

[6]. Shio Kumar Singh , M P Singh , and D K Singh "Routing Protocols in Wireless Sensor Networks - A Survey" International Journal of Computer Science \& Engineering Survey (IJCSES) Vol.1, No.2, November 2010. 